\title{
XXIX.
}

Aus der psychiatrischen Klinik zu Heidelberg. (Prof. Fürstner.)

\section{Beitrag zur pathologischen Anatomie der Gliose der Hirnrinde.}

(Nach einem auf der Versammlung der Südwestdeutschen Irrenärzte [30. October 1887] gehaltenen Vortrage.)

Von

\section{Dr. Buchholz,}

Assistent.

(Hierzu Taf. IX.)

In dem XVII. Bande dieses Archivs sind von Prof. Fürstner und Dr. Stühlinger in Rückblick auf einige ältere Beobachtungen vier Fäle einer eigenartigen Erkrankung des Centraluervensystems beschrieben und diesem Befunde die Bezeichnung "Gliose der Hirnrinde" beigelegt worden. Ein Gehirn, das Herr Prof. Fürstner mir zur Untersuchung za überlassen die Güte hatte, wies im Allgemeinen dieselben Veränderungen auf, bot jedoch-insofern noch einzelne interessante Befunde, als sich an demselben eine Reihe von Stellen auffinden liessen, die uns einen Einblick gewähren in das Verhältniss jener in der vorher erwähnten Arbeit bereits eingehend geschilderten circumscripten zu den mehr allgemeinen Veränderungen der krankhaft entarteten Rinde.

Auch dieses Gehirn zeigt in seinen vorderen Partien eine deutliche Atrophie - schmale, kammartige Gyri, breite und tiefe Sulci die sich jedoch ausserdem auch noch in besonders ausgesprochener Weise auf die der Insel benachbarten Windungszüge ausgebreitet hat.

Die Oberfläche aller dieser Gyri - der beiden Centralwindungen 
und Lobuli paracentrales, der Gyri recti und cinguli, der drei Stirnwindungen, der obersten Temporal- und der Inselwindungen - zeigt jedoch nicht die gewöhnliche, gleichmässige, glatte Beschaffenheit, sondern ist vielmehr mit einer Unzahl gerade noch sichtbarer bis stecknadelkopfgrosser Höckerchen von meist stumpfkegelförmiger Gestalt übersät. Einschnitte in diese Höckerchen liessen dieselben als solide Massen jedenfalls frei von makroskopisch wahrnehmbaren Hohlräumen erscheinen; das sie constitnirende Gewebe geht direct in die darunter gelegene Gehirnsubstanz über. An einer Stelle nun fübrte ein Einschnitt auf ein im grössten Durchmesser 11/2 Mrm. messendes, sich durch das Gefüge seines Gewebes gegen die Umgebung dentlich abhebendes Gebilde, das, wie eine eingehendere Untersuchung lehrte, durchaus jenen in der vorher citirten Arbeit erwähnten „Höhlen “ entspricht, zumal da auch hier bereits makroskopisch in ihrer Mitte ein winziger Hohlraum nachzuweisen war. Wenn ich nun auch weiterhin für dieses ganze Gebilde nach dem Vorgange von Fürstner und Stühlinger die Bezeichnung „Höhle" beibehalte, so möchte ich gleich hier bemerken, dass ich als das Wesentliche dieser Höhlen nicht den Hohlraum ansehe, sondern auch gleichartige Gebilde, selbst wenn sie keinen Hohlraum erkennen lassen, vorläufig, da wir noch keine passendere Bezeichnung besitzen, unter diesen Ausdruck subsumiren werde.

Es lag diese Höhle dicht unter der anscheinend nicht weiter veränderten Oberfläche eines kleinen, die Kante zwischen äusserer und innerer Fläche des rechten Stirnhirns überbrückenden Windungszuges, der mithin zu der ersten Stirnwindung zu rechnen wäre. Mit ibrem inneren Rande erreichte die Höhle die weisse Substanz, doch zog sich auch hier noch ein' schmaler Saum grauer Rindensubstanz, einen nach innen convexen Bogen beschreibend, um dieselbe herum. Bereits makroskopisch liess sich das die starke Wandung der Höhle bildende Gewebe als bindegewebige faserige Massen erkennen, die in einer zwar unregelmässigen, jedoch im Ganzen ungefähr kreisförmigen Contour die Höble gegen die Umgebung abschloss. Noch weitere makroskopisch sichtbare Höhlen aufzufinden, gelang mir trotz vieler Einschnitte nicht.

Was die weichen Hirnhänte anbetrifft, so möchte ich gleich hier, bevor ich mich der Beschreibung der mikroskopischen Gehirnbefunde zuwende, erwähnen, dass sie die Zeichen eines chronischen Entzündungsprocesses darboten, wie wir sie ja auch sonst z. B. bei der Dementia paralytica zu finden pflegen; makroskopisch: Trübung und Verdickung, mikroskopisch: starke Füllung der feinen und feinsten 
Gefässe, Auswanderung von Leucocythen, Vermehrung der Bindegewebszellen, Vermehrung und Verdichtung der sonstigen bindegewebigen Elemente.

Schnittpräparate aus jenen vorher aufgezählten, den vorderen Theilen des Gehirns entnommenen Partien zeigen, und zwar bereits für makroskopische Betrachtung bemerkbar, ganz allgemein eine stark verbreiterte, wenig tingirbare I. Schicht - Schicht der zerstrenten Rindenkörper von Meynert. Wie gesagt, kann man bereits ohne Zuhülfenahme des Mikroskops, besonders wenn man die Schnitte gegen das Licht bält, diese I. Schicht dentlich als einen hellen, die tiefer gelegenen Partien umgebenden, bald breiteren, bald schmäleren Sanm wahrnehmen; eine Veränderung, welche ja auch in jener älteren Arbeit eingehend beschrieben worden ist.

Dieselbe Uebereinstimmung in den Befunden ergab dann aber anch die weitere Untersuchung. Anch hier war es die oberste Rindenscbicht, welche die weitgehendsten Veränderungen aufwies, während die tieferen Lagen nur in geringerem Grade die Zeichen einer Erkrankung darboten, und zwar war die I. Schicht derartig verändert, dass sich innerhalb derselben an Stelle des normalen Gewebes ein bald mehr feines und weitmaschiges, bald mehr derbes und dichtes Fasernetz vorfand, zwischen welches gleichfalls, an Zahl je nach dem Orte wechselnd, immerhin jedoch in erheblich vergrösserter Menge, spinnenzellenartige Gebilde eingelagert waren.

Neben diesen Zellen zeigten sich ausserdem noch in dem Gewebe zerstreat, und zwar am zahlreichsten in der Nähe von Gefässen, ein Punkt, den ich später noch berühren werde, reichliche Rundzellen, die wohl für nichts anderes als ausgewanderte Lencocythen angesehen werden können. Zwischen diesen, so verschiedenen Zellformen konnten dann aber zahlreiche Uebergangsformen nachgewiesen werden, d. h. Zellen mit noch relativ grossem Kern and Protoplasmaleib und breitbasigen protoplasmareichen Fortsätzen. An vielen dieser Zellen liess sich noch ein Zusammenhang eines solchen mehr oder minder feinen Fortsatzes mit der Adventitialscheide eines Gefässes, dieser gewöhnlich keilförmig aufsitzend, nachweisen. Ob es sich nun aber in solchen Fällen wirklich um eine Ausstülpung der Adventitialscheide resp. eines ibrer Blätter handelt, wie es allerdings den Anschein hat, dürfte mit Sicherheit zu entscheiden, zur Zeit nicht möglich sein, besonders da die Möglichkeit, dass Fortsätze von Spinnenzellen an Gefässe heranwachsen, durchaus zugegeben werden muss; zumal da ja auch, abgesehen ron sonstigen Gründen die auch hier gar nicht so selten beobachtete Erscheinung, dass Spinnenzellen nicht nur mit 
einem, sondern mit mehreren Gefässen in Verbindung treten, wohl nur eine derartige Anffassung zulässt. Ohne Frage jedoch kann behauptet werden, dass diese sich so vielfach kreuzenden und verflechtenden Fortsätze der ihrer Zahl nach vermehrten Spinnenzellen es sind, welche der obersten Rindenschicht das Aussehen eines mehr oder minder feinen Fasernetzes mit eingelagerten Zellen verleihen.

Allerdings kommen hier wohl weniger die noch protoplasmareichen Fortsätze jener, ich möchte sagen, noch jungen Spinnenzellen in Betracht, als vielmehr jene Zellen, die bei einem minimalen Leibe änsserst lange und mehr derbere Fortsätze aussenden.

Ausser diesen noch deutlich als Ausläufer von Spinnenzellen erkennbaren Fasern, finden wir aber an einzelnen Stellen Faserzüge, die sich aus Fibrillen zusammensetzen, deren Caliber für die immerhin doch feinen Fortsätze von Spinnenzellen -, die protoplasmareichen können hierbei natürlich gar nicht in Frage kommen - zu stark ist und die ausserdem ein mehr derbes und starreres Aussehen besitzen. Derartige Faserzüge sind besonders stark in der Umgebung jener vorher erwähnten Höhle und verwandter, später noch zu beschreibender Gebilde anzutreffen. Nach der ganzen Art ihres Aussehens und dem Orte ihres Vorkommens würden wir aber diese Faserzüge nicht principiell von jenen Spinnebzellen zu trennen, sondern vielmehr nur als weitere Entwickelungsstadien ein und desselben Processes aufzufassen haben. An und für sich würde ja auch diese Umwandlung von Zellen der Bindegewebsgruppe in Fasern bei den vielfachen Analogien, z. B. Entwickelung des Narbengewebes kaum etwas Befremdendes an sich haben. Fine derartige Auffassung lässt uns darn aber auch eine Erklärng für die Entstehung jener bereits makroskopisch sichtbaren, später näher zu beschreibenden Bindegewebslagen der Höhlenwandung finden, deren Entwickelung sonst uns ganz unverständlich sein würde.

Ich habe vorhin erwähnt, dass die Vertheilung der Spinnenzellen über die erste Schicht eine ungleichmässige ist, dass sie an einzelnen Stellen dichtgedrängt bei einander liegen, an anderen mehr vereinzelt aufzufinden sind, wenn auch wohl keine Stelle so wenig Zellen, wie eine normale Rinde aufweist. Der Lieblingssitz dieser Zellen ist ohne Frage die Umgebung der Gefässe, und zwar finden wir hier vorherrschend jene mebr jungen Formen von Spinnenzellen und zwischen ihnen beinahe regelmässig noch eine grössere Anzahl von Rundzellen.

Was nun die Gefässe selbst anbetrifft, so waren dieselben vielfach in erheblichem Grade einem krankhaften Processe anheimgefallen. Auch in diesem Falle waren es ror Allem die Gefässe der 
obersten Rindenschicht, die die hauptsächlichsten Veränderungen aufwiesen. An allen Gefässen liess sich eine starke Anfüllung derselben mit rothen Blutkörperchen nachweisen, ausserdem eine Verdickung der Wandung und eine mehr minder starke Vermehrung der in letzterer gelegenen Kerne. Dabei waren die adventitiellen Scheiden der Gefässe mit Leucocythen erfüllt, von welchen einzelne das Bild der Auswanderung aus der Scheide in das umliegende Gewebe darboten. Während wir hier demnach an den Gefässen noch die Erscheinungen eines chronisch entzündlichen Processes vor uns haben, fallen uns vielfach andere Gefässe anf, die bereits die Zeichen eines weiteren destruirenden Processes darbieten. Hier ist von den einzelnen Schichten der Gefässwandung nichts mehr wahrzunehmen, die Lumina der Gefässe und adventitiellen Scheiden sind untergegangen, wir haben nur noch ein bald mehr homogenes, schlauchartiges Gebilde vor uns, bald einen Strang von zahlreichen, hauptsächlich parallel angeordneten, dicht bei einander liegenden Fasern. Dass es sich bei diesen Gebilden um Gefässrudimente handelt, lehren einmal die Bilder, die den Vebergang der einen in die andere Formation vermitteln, dann aber auch die Verlaufsrichtung dieser bindegewebigen Züge und ihr Verhalten gegen die Umgebung. Auch ihre nächste Umgebung ist durch die grosse Nenge von Spinnenzellen ausgezeichnet, die nicht selten auch noch mit diesen Ceberresten von Gefässen durch Fortsätze in Verbindung treten. Wenn dem aber so ist, so können wir den ganzen Process nur als einen solchen auffassen, der za einem Untergange von Gefässen führt. Damit steht nun freilich im Widerspruch, dass man in dem einzelnen Gesichtsfelde auffallend viel Gefässe sieht. Dieser Widerspruch dürfte jedoch ơnrchaus nur scheinbar und dadurch bedingt sein, dass, bei der starken Atrophie des Gehirns, die Gefässe durch den Schwond des dazwischen liegenden Gewebes rein mechanisch einander näher gerückt worden sind. Dass aber die Atrophie trotz der beobachteten Volumenzunahme der obersten Rindenschicht eine im Ganzen doch recht erhebliche war, geht wohl deutlich schon aus dem oben geschilderten makroskopischen Befunde hervor.

Ausser diesem mehr allgemeineren, die Rinde des Vorderhirns betreffenden Befunde, ziehen dann aber an Schnitten besonders jene mehr circumscripten Veränderungen die Aufmerksamkeit auf sich, die Durchschnitte jener vorher erwähnten Tubera und Höhlen.

Wie oben gesagt, erscheint die oberste Rindenschicht verbreitert, jedoch ist diese Verbreiterung keine gleichmässige, wir sehen vielmehr, 
dass das diese Schicht bildende Gewebe in unregelmässiger Weise in die tieferen Partien hineinwuchert. $O b$ dabei an diesen Stellen die ursprünglichen Gewebselemente zu Grunde gelien oder einfach bei Seite gedrängt werden, ist mit Sicherbeit nicht zu entscheiden, jedoch ist es am wahrscheinlichsten, dass das eine neben dem anderen vorkommt. Wie dem auch sei, jedenfalls finden wir, besonders auffallend bei schwächeren Vergrösserungen, an Stelle jener normaler Weise scharfen und gerade verlaufenden Grenzlinie zwischen I. und II. Schicht einen vielfach gewundenen, bald mehr bald weniger weit in convexem Bogen nach innen vordringenden Contour. In ähnlicher Weise unregelmässig und ausgezackt ist aber auch die äussere Grenzlinie, indem wir hier die äussere Umgrenzung der Durchschnitte jener oben geschilderten Tubera vor uns haben. Diese weisen in der grössten Nehrzahl in ibrem Bau kẹine wesentliche Verschiedenheit gegen die oben besprochenen allgemeinen Veränderungen auf, so dass es sich also schliesslich nur um ein Hinauswachern dieses veränderten Gewebes über die ursprüngliche Oberfläche bandeln würde. In ähnlicher Weise können wir uns aber wohl auch die nach innen vorgeschobenen Zapfen entstanden denken.

Immerhin jedoch lassen diese Tubera noch gewisse Besonderheiten in ihrem Bau erkennen. So finden wir hier relativ wohl wenig zellige Elemente, und zwar beinahe nur jene mit langen mehr derben Fortsätzen ausgestatteten Spinnenzellen, daneben aber desto zahlreicher jene mehr rein faserartigen Gebilde, die hier im Allgemeinen eine gewisse gleichmässige Anordnung zeigen, und zwar sehen wir dieselben hier einmal dicht aneinander gedrängt in büschelförmiger Weise die Oberfläche überragen, andere Male in gleichmässig paralleler Anordnung nach aussen ziehen, nur von wenigen dazu senkrecht verlaufenden Faserzügen durchbrochen. Häufig macht sich dann in der Mitte dieser Höckerchen ein Gefäss resp. Gefässrudisnent geltend, und wir werden wohl nicht fehl gehen, wenn wir annehmen, dass dieses ursprünglich den Ausgangspunkt für die Bildung dieser Excrescenzen abgegeben hat.

Neben diesen Tubera fallen aber vereinzelt andere auf, die einen ganz anderen eigenartigen Bau zeigen. Hier finden wir nichts mehr von jenem feinen, den allgemeinen Veränderungen entsprechendem Fasernetze, vielmehr ist seine Stelle von dicken Bindegewebszügen eingenommen, und zwar werden dieselben aus einzelnen bald mehr derben, bald mehr feinen und anscheinend weicheren Bindegewebslamellen zusammengesetzt, welchen langgestreckte, häufig beinahe stäbchenförmige Kerne eingelagert sind, neben welchen allerdings 
auch immer noch eine Anzahl rundkerniger Gebilde zar Beabachtung kommen.

Ueber die Lagerung der einzelnen Bindegewebslamellen wäre hier noch zu bemerken, dass dieselben im Allgemeinen zwar vielfach gewellt sind, im Ganzen und Grossen doch eine concentrische Anordnung zeigen, nnd zwar sind es die derberen, jedenfalls älteren Bindegewebszüge, welche der Peripherie der einzelnen Tubera zunächst gelagert sind (Fig. I.). Diese Tubera gehen auch nicht so ganz unvermittelt in das daruntergelegene Gewebe über, sondern grenzen sich eben durch diese derben Fasermassen deutlich gegen dasselbe ab. Dieses selbst ist nun aber keineswegs normal, sondern zeigt die vorher geschilderten allgemeinen Verändernngen gerade in einem besonders starken Masse, d. b. wir finden hier ein relativ derbes Fasernetz, das nicht selten an der Grenze der Tubera eine arcadenartige Anordnung zeigt.

Ganz dieselben Veränderungen fivden wir aber auch in der Umgebung der oben makroskopisch beschriebenen Höhle, nur dass wir hier diese arcadenartigen Bindegewebszüge, wohl entsprechend der bedentenderen Grösse und - um es hier gleich vorwegzunehmen wohl auch dem bedeutenderen Alter der Höhle viel weiter in das umliegende Gewebe hinein verfolgen können. Besonders interessant war hier, dass sich aus den Arcaden heraus zwei Faserzüge entwickeln lassen, die durch minder verändertes Gewebe hindurch von beiden Seiten der Höhle aus der Rinde zustrebten, in welchen Gebilden wir wohl ohne Frage Gefässrudimente zu erblicken baben. Der zwischen diesen beiden Zügen einerseits dem oberen Rande der Höhle und der freien Oberfläche andererseits gelegene Strich zeichnet sich durch eine besonders feine Zeichnung aus, indem sich daselbst ein äusserst zierliches Gitterwerk von Spinnenzellen, die durch ihre Fortsätze miteinander in Verbindung stehen, präsentirt*).

Was nun die Höhle selbst anbetrifft, so zeigte sich ihre Wandung aus ziemlich derben Gewebslagen zusammengesetzt, über deren eigenthümliche Lagerung wohl die Zeichnung (Fig. 2) die beste Aufklärung geben dürfte. Erwähnen möchte ich nur, dass auch hier an der Peripherie die stärksten Faserzüge anzutreffen sind, während in den central gelegenen Partien ein weicheres und welligeres Gewebe

*) In der Zeichnung Fig. 2 bei a konnten diese Verhältnisse der gewählton schwachen Vergrösserung halber ( $Z_{\theta}$ iss, Oc. 2 Obj. a) leider nicht zum Ausdruck gebracht werden. 
vorherrscht, in welchem neben zahlreichen Kernen auch noch einzelne Gefässrudimente anzutreffen sind.

In der Mitte der Hoble - in dem gezeichneten Schnitte ist derselbe nicht mitgetroffen - befand sich ein kleiner Hohlraum von unregelmässiger, nicht scharfer Umgrenzung, der eine geringe Menge von Flüssigkeit enthielt, über deren Beschaffenheit ein Urtheil abzugeben, mir unmöglich ist. An einzelnen Präparaten waren hier noch, jedenfalls Reste des ursprünglichen Inhalts, rundliche, sich mit Carmin, Hämatoxylin und Rubin stark tingirende tropfenartige Elemente anzutreffen.

Es musste sich nuu, nachdem die Untersuchung zu diesem Resultate geführt hatte, von selbst die Frage aufdrängen: stehen sich diese zuletzt erwähnten Befunde und jene mehr allgemeinen Veränderungen unvermittelt gegenüber oder sind sie etwa Producte desselben pathologischen Processes? Es finden sich nun eine Reihe von Präparaten - und deshalb gerade habe ich die Resultate der Untersuchung dieses Gehirns der Mittheilung werth erachtet - die nur eine Erklärung im letzteren Sinne zulassen. Und zwar sehen wir an diesen Präparaten, dass von einer centralen, innerhalb der I. Schicht gelegenen Partie, die aus einigen mit Kernen besetzten Bindegewebsbälkchen gebildet wird (Fig. III. bei a), eine Reihe von im Allgemeinen radiärgerichteten, ziemlich derben Fasern ausgeht, die dann in einer Masse circulär angeordneter Fasern verschwinden. Letztere sind derber, als etwa die Fortsätze von Spinnenzellen, liegen dicht neben einander und zeigen in ihrer Anordnung einen ganz regelmässigen Typus, indem sie in bogenförmigen Zügen die mittlere Partie umgeben. In dieser selbst jedocb erkennen wir aus dem Vergleiche mit anderen Präparaten obne Mähe die Reste eines degenerirten Gefässes, von dessen ursprünglicher Wandung allerdings nichts mehr zu seben ist. Wir haben vielmehr nur noch den Durchschnitt eines stärkeren Faserzuges, wie diese ja auch vorher bereits geschildert worden sind, vor uns, dem eine Reihe von Spinnenzellen angelagert ist, als deren Fortsätze wir den grössten Theil jener radiär angeordneten Fasern zu betrachten hätten. Dass ich die circulär angeordneten Easerzüge äbrigeus den oben beschriebenen in der Umgebung der Tubera und Höhle vorzufindenden Fasern für gleichwerthig erachte und auch in ihnen nichts anders erblicken kann, als in besonderer Weise umgewandelte Zellen, brauche ich wohl nur zu erwähnen.

Wir würden dann aber in den Grundzügen in diesen Bildern bereits die Anfänge jener Tubera- und Höhlenbildung vor uns haben, da die Annahme, dass die bereits hier schon recht derben Fasern in 
dem weiteren Verlaufe sich zu jenen massigen Bindegewebslagen umbilden, nicht als unberechtigt erscheinen dürfte. Die Lage dieser central gelegenen Partien und die Richtung, in welcher sich dann die Mehrzahl der Fasern an dieselbe anlegt, wird entscheidend sein dafür, ob $\in$ s schliesslich zu der Bildung eines Tubers oder einer innerhalb der Rinde gelegenen Höhle kommt.

Ebenso wie in den früheren Fällen zeigte auch dieses Gehirn im Uebrigen nur geringe Veränderungen. Die Ganglienzellen der tieferen Hirnrindenschichten boten keinen sicheren pathologischen Befund, nur schienen sie an den Stellen, an welchen die Wucherungen der ersten Schicht in die Tiefe vorgedrungen war, zur Seite gedrängt worden zu sein, wenigstens fanden sich häufig Zellen, die mit ihrer Spitze nach einer Seite hin abwichen. Wahrscheinlich ist es allerdings, dass die Zahl der Ganglienzellen resp. der in der Rinde und den Markleisten vorhandenen Nervenfasern abgenommen hat, da sonst, zumal bei der Vergrösserung der ersten Schicht. für die so deutliche Atrophie keine Erklärung zu finden wäre. Zur Untersuchung auf den seventuellen Schwund der Associationsfasern wurden die Schnitte mit der Weigert'schen Kupfer-Hämatoxylin-Methode behandelt, wobei sich jedoch weder in den Markleisten, noch in den in der Rinde gelegenen Tangentialfasersystem ein deutlicher Schwund nachweisen liess. Allerdings zeigten die letzterwähnten Fasern nicht die gewöhnliche, mehr gleichmässig parallele Anordnung und Dichte, doch werden wir bei der Beurtheilung dieses Befundes doch auch die Auflockerung und Verbreiterung der ersten Rindenschicht in Rechnung ziehen müssen. An den Stellen freilich, an welchen sich rein bindegewebige Tubera und Höblen etablirt haben, sind die Nervenfasern, wie das ja auch nicht anders zu erwarten war, vollständig verschwunden, doch lassen sie sich anch hier noch weit in das so hochgradig veränderte Gewebe hinein verfolgen.

Ein sicheres Urtheil habe ich demnach nicht darüber gewinnen können, $o b$ es in diesem Falle wirklich nicht zu einem theilweisen Untergange - abgesehen von dem zuletzt erwähnten engumgrenzten Befunde - dieses Fasersystems gekommen ist, besonders da mir nur eine Art der Untersuchnng - die Exuer'sche Osmiumsäure-Methode war bei den nicht frischen Präparaten ausgeschlossen - zu Gebote staud.

Die Optici zeigten, auch hierin stimmt der Befund mit den früheren Untersuchungen überein, eine deutliche, ziemlich hochgradige, auf beiden Seiten gleichmässige Atrophie. $O b$ es anch in diesem Falle zu einer Degeneration der Hinterstränge des Rückenmarks ge- 
600 Dr. Buchholz, Beitrag zur pathol. Anatomie der Gliose der Hirnrinde.

kommen ist, worauf ja die Opticusdegeneration in einem gewissen Sinne hinweist, habe ich leider nicht constatiren können, da mir das Rückenmark aus äusseren Gründen nicht mehr zur Untersuchung zu Gebote stand.

\section{Erklärung der Abbildungen (Taf. IX.)}

Fig. 1. Extracorticaler Höcker. Hämatoxylin-Präparat. (Zeiss, Oc. 2. Objec. A.)

Mit gütiger Erlanbniss des Herrn Prof. Fürstner habe ich für diese Zeichnung ein Präparat von einem jener älteren Fälle benutzt, da dieses in besonders deutlicher Weise die Veränderungen zum Ausdruck brachte.

Fig. 2. Höhle innerhalb der I. Frontalwindung. Hämatoxylin-RubinPräparat. (Zөiss, Oc. 2. Objec. a ${ }_{3}$.)

Fig. 3. Schnitt ans der vorderen rechten Centralwindung. Borax-Carmin-Präparat. (Zeiss, Oc. 2. Objec. DD.) 


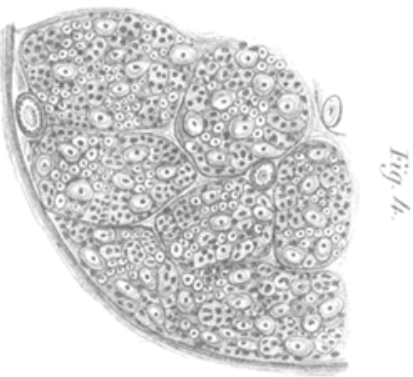

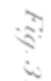
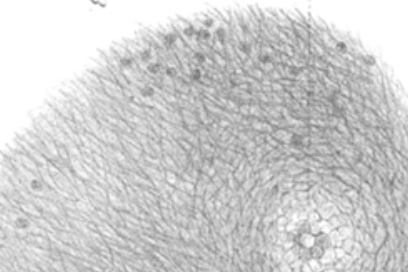

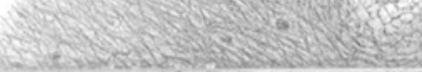

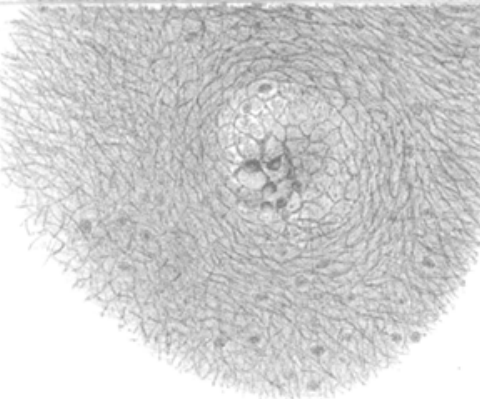

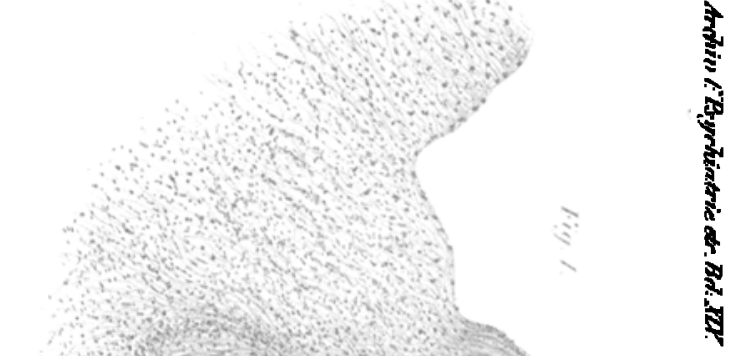

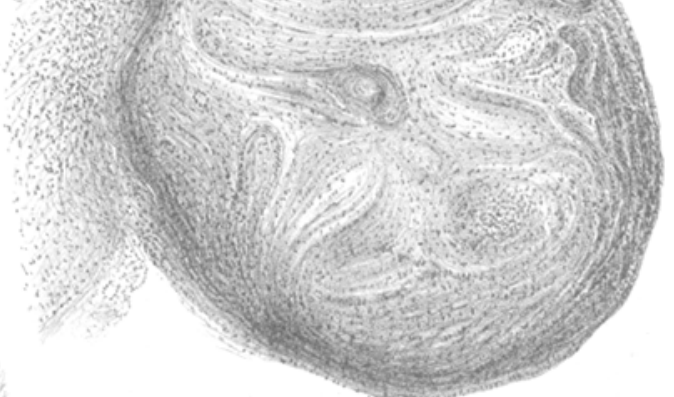

and 7 (n)

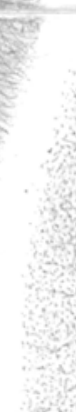

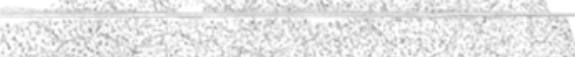
(1)

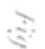

? 